\title{
Comment on "Relation between scattering amplitude and Bethe-Salpeter wave function in quantum field theory"
}

\author{
Sinya Aoki, ${ }^{1}$ Takumi Doi, ${ }^{2,3}$ Tetsuo Hatsuda, ${ }^{3,2}$ and Noriyoshi Ishiii ${ }^{4}$ \\ ${ }^{1}$ Center for Gravitational Physics, Yukawa Institute for Theoretical Physics, Kyoto University, \\ Kyoto 606-8502, Japan \\ ${ }^{2}$ RIKEN Nishina Center, RIKEN, Saitama 351-0198, Japan \\ ${ }^{3}$ RIKEN Interdisciplinary Theoretical and Mathematical Sciences Program (iTHEMS), RIKEN, \\ Wako 351-0198, Japan \\ ${ }^{4}$ Research Center for Nuclear Physics (RCNP), Osaka University, Osaka 567-0047, Japan
}

(Received 12 February 2018; published 28 August 2018)

\begin{abstract}
We invalidate the arguments given in [T. Yamazaki and Y. Kuramashi, Phys. Rev. D 96, 114511 (2017)] over the HAL QCD method for hadron-hadron interactions on the lattice. We also pose questions on the practical usefulness of the method proposed in this reference.
\end{abstract}

DOI: 10.1103/PhysRevD.98.038501

In a recent article [1], Yamazaki and Kuramashi present a theoretical analysis on the "relation between scattering amplitude and Bethe-Salpeter wave function in quantum field theory." This subject is important for hadron-hadron interactions in lattice QCD simulations and is closely related to the physics of multihadron systems such as the exotic resonances and the atomic nuclei.

In the present comment, we show that the arguments in Ref. [1] over the HAL QCD method [2-5] for hadronhadron interactions can be invalidated on the basis of the previously published works by the present authors; in particular, Sec. 2, Sec. 3.1, Sec. 3.2, Appendix A and Appendix B of [2], as well as Sec. I of [3].

Let us consider the interaction between bosons with identical mass $m$ as analyzed in [1]. The basic idea of the HAL QCD method is to define the energy-independent and nonlocal potential $U\left(\mathbf{r}, \mathbf{r}^{\prime}\right)$ [2-5] from the Nambu-BetheSalpeter (NBS) wave function $\phi_{\mathbf{k}}(\mathbf{r})$ below the inelastic threshold $\left(|\mathbf{k}|<\mathrm{k}_{\mathrm{th}}\right)$ as

$$
\begin{aligned}
\left(\nabla^{2}+\mathbf{k}^{2}\right) \phi_{\mathbf{k}}(\mathbf{r}) & =m V(\mathbf{r} ; \mathbf{k}) \phi_{\mathbf{k}}(\mathbf{r}) \\
& =m \int d^{3} r^{\prime} U\left(\mathbf{r}, \mathbf{r}^{\prime}\right) \phi_{\mathbf{k}}\left(\mathbf{r}^{\prime}\right)
\end{aligned}
$$

The primary confusion of Ref. [1] originates from a claim that $V(\mathbf{r} ; \mathbf{k})$ in Eq. (1) is replaced by $V(\mathbf{r} ; \mathbf{q})$ even for $\mathbf{q} \neq \mathbf{k}$ in the HAL QCD method. Such a replacement

Published by the American Physical Society under the terms of the Creative Commons Attribution 4.0 International license. Further distribution of this work must maintain attribution to the author(s) and the published article's title, journal citation, and DOI. Funded by SCOAP ${ }^{3}$. however has never been introduced in the HAL QCD method. The correct mathematical relation between $U\left(\mathbf{r}, \mathbf{r}^{\prime}\right)$ and $V(\mathbf{r} ; \mathbf{k})$ has been shown to be [see Eq. (2.3) in [2] and Eq. (7) in [3] ]

$$
U\left(\mathbf{r}, \mathbf{r}^{\prime}\right)=\sum_{|\mathbf{k}|<\mathrm{k}_{\mathrm{th}}} V(\mathbf{r} ; \mathbf{k}) \phi_{\mathbf{k}}(\mathbf{r}) \bar{\phi}_{\mathbf{k}}\left(\mathbf{r}^{\prime}\right)
$$

where $\bar{\phi}_{\mathbf{k}}\left(\mathbf{r}^{\prime}\right)$ are dual basis functions associated with the nonorthonormal basis functions $\phi_{\mathbf{k}}\left(\mathbf{r}^{\prime}\right)$, with the property, $\int d^{3} r \bar{\phi}_{\mathbf{k}}(\mathbf{r}) \phi_{\mathbf{q}}(\mathbf{r})=\delta_{\mathbf{k}, \mathbf{q}}$. Equation (2) represents a clear connection between the $\mathbf{k}$-dependent local-potential $V(\mathbf{r} ; \mathbf{k})$ and the $\mathbf{k}$-independent nonlocal potential $U\left(\mathbf{r}, \mathbf{r}^{\prime}\right)$.

In the practical applications of the HAL QCD method [2-5], the expansion of $U\left(\mathbf{r}, \mathbf{r}^{\prime}\right)$ in terms of its nonlocality is employed according to the well-known method of derivative expansion [6,7],

$$
U\left(\mathbf{r}, \mathbf{r}^{\prime}\right)=\sum_{\mathbf{n}} V_{\mathbf{n}}(\mathbf{r}) \nabla_{\mathbf{r}}^{\mathbf{n}} \delta^{(3)}\left(\mathbf{r}-\mathbf{r}^{\prime}\right),
$$

with $\nabla_{\mathbf{r}}^{\mathbf{n}} \equiv \nabla_{r_{x}}^{n_{x}} \nabla_{r_{y}}^{n_{y}} \nabla_{r_{z}}^{n_{z}}$. (Here we have not assumed any spatial symmetry.) The $\nabla$ dependence is historically called the velocity dependence, since it is the origin of the spinorbit term, the quadratic spin-orbit term, etc., in the case of the nuclear force. (See the original articles, Refs. [6,7], and Appendix B of Ref. [2].) Possible systematic errors associated with the truncation of this expansion can be estimated through order-by-order analysis using lattice QCD data [8-10].

In Ref. [1], there is a confusion on the number of degrees of freedom. First of all, $U\left(\mathbf{r}, \mathbf{r}^{\prime}\right)$ is $\mathbf{k}$ independent from

\footnotetext{
${ }^{1}$ For detailed analysis with inelastic channels, see Ref. [3].
} 
Eq. (2), so that $V_{\mathbf{n}}(\mathbf{r})$ is $\mathbf{k}$ independent from Eq. (3). Secondly, $\mathbf{n}$ (" $i$ " in the notation of Ref. [1]) is a threedimensional vector, so that there are sufficient degrees of freedom in the right-hand side of Eq. (3) to represent the nonlocality of $U\left(\mathbf{r}, \mathbf{r}^{\prime}\right)$. In fact, as shown explicitly in Sec. II.D of Ref. [9], the inversion of Eq. (3) reads

$$
V_{\mathbf{n}}(\mathbf{r})=\int d^{3} \mathbf{r}^{\prime} U\left(\mathbf{r}, \mathbf{r}^{\prime}\right) \frac{\left(\mathbf{r}^{\prime}-\mathbf{r}\right)^{\mathbf{n}}}{\mathbf{n} !}
$$

with $\left(\mathbf{r}^{\prime}-\mathbf{r}\right)^{\mathbf{n}} / \mathbf{n} ! \equiv \prod_{\ell=x, y, z}\left(r^{\prime}-r\right)_{\ell}^{n_{\ell}} / n_{\ell} !$. This implies $V_{\mathbf{n}}(\mathbf{r})$ is manifestly $\mathbf{k}$ independent. Here we note that the above integral is expected to be convergent below the inelastic threshold, since there are no massless particles as asymptotic fields in QCD with physical quark masses.

In Ref. [1], there is a statement that "The problem in this expansion becomes manifest in the practical determination of $V_{i}(x)$. The simplest example is the determination of the leading term $V_{0}(x)$, which is approximated by $h(x ; k) / \phi(x ; k)$. We find that it should contain the contributions of $O\left(k^{2 n}\right)(n \geq 0)$ from the higher order terms of the velocity expansion in order to properly describe the $k$ dependence of $h(x ; k)$." This statement originates from a confusion of the systematics from the truncation of the derivative expansion with the $k$ dependence. To make this point clear, let us consider a hypothetical nonlocal potential $U^{(2)}$ which has only two terms in the right-hand side of Eq. (2),

$$
U^{(2)}\left(\mathbf{r}, \mathbf{r}^{\prime}\right)=\left(V_{0}(\mathbf{r})+V_{2}(\mathbf{r}) \nabla^{2}\right) \delta^{(3)}\left(\mathbf{r}-\mathbf{r}^{\prime}\right) .
$$

Suppose we have two NBS wave functions at different momentums, $\phi_{\mathbf{k}}(\mathbf{r})$ and $\phi_{\mathbf{k}^{\prime}}(\mathbf{r})$. Then, we can reconstruct the potentials, $V_{0}$ and $V_{2}$, as

$$
\left(\begin{array}{l}
V_{0}(\mathbf{r}) \\
V_{2}(\mathbf{r})
\end{array}\right)=\frac{1}{D}\left(\begin{array}{cc}
\nabla^{2} \phi_{\mathbf{k}^{\prime}}(\mathbf{r}) & -\nabla^{2} \phi_{\mathbf{k}}(\mathbf{r}) \\
-\phi_{\mathbf{k}^{\prime}}(\mathbf{r}) & \phi_{\mathbf{k}}(\mathbf{r})
\end{array}\right)\left(\begin{array}{c}
h(\mathbf{r} ; \mathbf{k}) \\
h\left(\mathbf{r} ; \mathbf{k}^{\prime}\right)
\end{array}\right),
$$

with $D \equiv m\left(\phi_{\mathbf{k}}(\mathbf{r}) \nabla^{2} \phi_{\mathbf{k}^{\prime}}(\mathbf{r})-\phi_{\mathbf{k}^{\prime}}(\mathbf{r}) \nabla^{2} \phi_{\mathbf{k}}(\mathbf{r})\right)$. In this simple example, $V_{0}(\mathbf{r})$ and $V_{2}(\mathbf{r})$, which are $\mathbf{k}$ independent by definition, reproduce the exact value of the phase shift $\delta(\mathbf{k})$ at all $\mathbf{k}$ below the inelastic threshold. In more realistic cases with higher order derivative terms in Eq. (2), the phase shift $\delta(\mathbf{k})$ calculated from $V_{0,2}$ as constructed in Eqs. (6) is exact at $\mathbf{q}=\mathbf{k}$ and $\mathbf{k}^{\prime}$, and is only approximate at other q. How accurate at other $\mathbf{q}$ can be checked if more NBS wave functions for different momentums are available. In practice, the time-dependent HAL QCD method based on the Euclidean-time $(t)$ dependence of the hadronic correlation function is a useful equivalent method to treat those states with different momentums simultaneously, as demonstrated in [8].

In Ref. [1], there is also a statement that "Therefore, a smearing of the interpolating operator in the BS wave function gives a different scattering amplitude from the one obtained from the fundamental relation, which depends on the smearing function $s(x)$." As already shown explicitly in Sec. II. D of [9], this statement is mathematically incorrect. To clarify the source of this error, let us start with the wellknown formula [11]

$$
\phi_{\mathbf{k}}(\mathbf{r})=C_{\phi}(k) e^{i \mathbf{k} \cdot \mathbf{r}}+\int \frac{d^{3} p}{(2 \pi)^{3}} \frac{H_{\phi}(p ; k)}{p^{2}-k^{2}-i \epsilon} e^{i \mathbf{p} \cdot \mathbf{r}},
$$

where the NBS wave function $\phi_{\mathbf{k}}(\mathbf{r})$ is defined by

$$
\phi_{\mathbf{k}}(\mathbf{r}) \equiv\left\langle 0\left|\pi_{1}(\mathbf{r} / 2) \pi_{2}(-\mathbf{r} / 2)\right| \hat{\pi}_{1}(\mathbf{k}) \hat{\pi}_{2}(-\mathbf{k}) ; \text { in }\right\rangle,
$$

with an interpolating operator of the $i$ th scalar particle $\pi_{i}(i=1,2)$, and $C_{\phi}(k)$ being a normalization factor. The on-shell amplitude is related to the phase shift as $H_{\phi}(k ; k) / C_{\phi}(k)=4 \pi e^{i \delta(k)} \sin \delta(k) / k$.

If we use the smeared NBS wave function $\tilde{\phi}_{\mathbf{k}}(\mathbf{r})$ with a smearing function, $s(\mathbf{r})$, acting e.g., on one of the operators in Eq. (8), one easily obtains

$$
\frac{H_{\phi}(k ; k)}{C_{\phi}(k)}=\frac{H_{\tilde{\phi}}(k ; k)}{C_{\tilde{\phi}}(k)}=\frac{4 \pi}{k} e^{i \delta(k)} \sin \delta(k),
$$

with $C_{\tilde{\phi}}(k) / C_{\phi}(k)=\int d^{3} r s(\mathbf{r}) e^{-i \mathbf{k} \cdot \mathbf{r}}$ by a simple change of variable [see Eq. (19) in [9] ]. Thus the smearing does not affect the observable as long as the correct normalization is taken into account. Note that the situation is the same even for composite sink operators as long as they are "almostlocal operator fields" defined by Haag in Ref. [12]. ${ }^{2}$

Some additional comments are in order here.

First, it is stated in Ref. [1] that "Although we call it as the effective potential here, the relation between this quantity and the potential in quantum mechanics is not trivial; the former is the reduced BS wave function normalized by the BS wave function being manifestly momentum dependent, while the latter is defined to be momentum independent in principle. This is an essential difference between relativistic quantum field theory and nonrelativistic quantum mechanics." This statement is inaccurate. The difference between local (momentumdependent) and nonlocal (momentum-independent) potentials does not have direct correspondence to the difference between nonrelativistic quantum mechanics and relativistic quantum field theory. There are numerous examples where nonlocal (momentum-independent) potentials appear in nonrelativistic quantum mechanics, especially for scattering problems of composite objects such as the atoms and atomic nuclei.

\footnotetext{
${ }^{2}$ See also a brief summary in Appendix A of [2] on the Nishijima-Zimmermann-Haag reduction formula for the scattering of composite particles.
} 
Secondly, the so-called fundamental relation [Eq. (11) in Ref. [1]]

$$
-\int d^{3} x h(x ; k) e^{-i \mathbf{k} \cdot \mathbf{x}}=\frac{4 \pi}{k} e^{i \delta(k)} \sin \delta(k)
$$

cannot provide an alternative method to determine the phase shift $\delta(k)$ in lattice QCD. Indeed, this equation is nothing but Eq. (9), so that the independent determinations of the normalization $C_{\phi}(k)$ and the momentum $k$ on the lattice are necessary. ${ }^{3}$ If $k$ is known, however, the phase shift $\delta(k)$ can be obtained directly from Lüscher's finite volume formula [13] without recourse to Eq. (10). Therefore, the above relation gives at most a consistency

\footnotetext{
${ }^{3}$ In contrast, $U\left(\mathbf{r}, \mathbf{r}^{\prime}\right)$ in Eq. (1) does not have such normalization issue due to the cancellation of $C_{\phi}(k)$ in both sides [2].
}

check for the determination of $k$, but does not provide an alternative method to obtain $\delta(k)$. Moreover, in the case that $k^{2}<0$ in the finite volume, the Fourier transformation of $h(x ; k)$ must be analytically continued to the pure imaginary $k$ by the numerical integral, which may introduce uncertainties due to statistical errors of $h(x ; k)$ at large $x$. These comments apply to a recent paper [14] too.

\section{ACKNOWLEDGMENTS}

This work is supported in part by a priority issue (elucidation of the fundamental laws and evolution of the Universe) to be tackled by using Post " $K$ " Computer, and by Joint Institute for Computational Fundamental Science (JICFuS) and MEXT Grant-in-Aid for Scientific Research (JP15K17667, JP16H03978, JP18H05236). T.D. and T.H. were partially supported by the RIKEN iTHEMS Program.
[1] T. Yamazaki and Y. Kuramashi, Phys. Rev. D 96, 114511 (2017).

[2] S. Aoki, T. Hatsuda, and N. Ishii, Prog. Theor. Phys. 123, 89 (2010).

[3] S. Aoki, B. Charron, T. Doi, T. Hatsuda, T. Inoue, and N. Ishii, Phys. Rev. D 87, 034512 (2013).

[4] N. Ishii, S. Aoki, T. Doi, T. Hatsuda, Y. Ikeda, T. Inoue, K. Murano, H. Nemura, and K. Sasaki (HAL QCD Collaboration), Phys. Lett. B 712, 437 (2012).

[5] S. Aoki et al. (HAL QCD Collaboration), Prog. Theor. Exp. Phys. 2012, 01A105 (2012).

[6] R. Tamagaki and W. Watari, Prog. Theor. Phys. Suppl. 39, 23 (1967).
[7] S. Okubo and R. E. Marshak, Ann. Phys. (N.Y.) 4, 166 (1958).

[8] T. Iritani (HAL QCD Collaboration), EPJ Web Conf. 175, 05008 (2018)

[9] D. Kawai et al., Prog. Theor. Exp. Phys. 2018, 043 B04 (2018).

[10] T. Iritani et al. (HAL QCD Collaboration), arXiv:1805.02365.

[11] C. J. D. Lin, G. Martinelli, C. T. Sachrajda, and M. Testa, Nucl. Phys. B619, 467 (2001).

[12] R. Haag, Phys. Rev. 112, 669 (1958).

[13] M. Lüscher, Nucl. Phys. B354, 531 (1991).

[14] Y. Namekawa and T. Yamazaki, Phys. Rev. D 98, 011501 (2018). 